\title{
The effect of statin therapy on coronary atherosclerosis as assessed by computed tomography
}

\author{
Darryl Wan $\mathrm{MD}^{1}$, A. Yashar Tashakkor $\mathrm{MD}^{1}$, Jonathon Leipsic $\mathrm{MD}^{2}$, Paolo Raggi MD³ , G.B. John Mancini MD ${ }^{1}$
}

Wan D, Tashakkor AY, Leipsic J, et al. The effect of statin therapy on coronary atherosclerosis as assessed by computed tomography. Curr Res Cardiol 2016;3(4):121-27.

OBJECTIVES: The effect of statin therapy on coronary artery calcification is unclear. Early studies suggested a slower rate of coronary artery calcium score (CACS) progression, but recent prospective trials have failed to show this benefit. Recent studies have explored the use of Cardiac Computed Tomography Angiography (CCTA) to characterize plaque features. We provide a systematic review of available literature documenting the effects of statin therapy on the progression of CACS and non-calcium-based indices.

METHODS: A systematic search was performed from January 1, 1980 to April 28, 2016 using these databases: Cochrane Database, ACP Journal Club, Health Technology Assessment, Embase, NHS Economic Evaluation Database, Ovid MEDLINE, Health and Psychosocial Instruments. English language publications that serially measured relationships between statin therapy and CACS or non- calcium-based indices were included. Case reports, reviews and meta-analyses were excluded. Data regarding progression of calcium and non-calcium-based indices were extracted and analyzed.

RESULTS: 2159 articles were retrieved for screening. Of these, 22 met pre-defined inclusion criteria; 9 were randomized controlled trials and 13 observational studies. Observational studies did not consistently demonstrate a reduction in the progression of CACS with statin therapy. No randomized trial demonstrated convincing evidence that statin therapy reduces the progression of CACS. Limited randomized trials of CCTA suggest that statin therapy may reduce non-calcified plaque volume, but increase dense calcium volume.

CONCLUSION: Based on studies using statins, serial assessment of non calcified plaque volume, but not CACS, may be useful for the assessment of medical interventions with postulated effects on progression or regression of atherosclerosis.

Key Words: Coronary artery calcium; CT angiography; Statins
Coronary artery calcium $(\mathrm{CAC})$ is a non-invasive marker of atherosclerosis (1-5). In several large-scale studies, coronary artery calcium scoring (CACS) has been shown to add prognostic value in predicting cardiovascular events when added to traditional risk stratification such as the Framingham score $(1,6-8)$. The 2010 ACCF/AHA Guidelines have advocated selective use of CACS for cardiovascular risk assessment in asymptomatic patients considered at intermediate risk through traditional assessment (9).

Statin therapy is an essential tool in the primary and secondary prevention of cardiovascular disease (10-17). Several trials have demonstrated that statins can induce regression of coronary atherosclerosis measured by intravascular ultrasonography (IVUS) in patients treated with high-intensity statin therapy $(18,19)$. Other studies have suggested that statin therapy promotes atheroma calcification, thereby stabilizing plaque $(20,21)$. Whether regression of atherosclerosis by statins can be assessed using serial CACS or CCTA remains controversial.

Early observational studies (22-24) suggested that statin therapy had the potential to slow CACS. However, subsequent randomized controlled trials (25-29) failed to confirm this. And more recent studies of statin effects on CACS in special populations such as Systemic Lupus Erythematosus (SLE) (30) and chronic kidney disease (CKD) patients are also controversial due to small study populations (31).

Recent studies have also investigated plaque changes as measured by non-calcium-based indices of coronary artery disease on CCTA to further characterize features of coronary plaques. Whether these measurements are useful for serial assessments remains to be seen. Accordingly, the purpose of this review is to elucidate the effect of statin therapy on CACS and noncalcium-based indices of coronary artery disease progression through a systematic review.

\section{METHODS}

Data sources and searches

A systematic search was performed on the following databases for articles published from January 1, 1980 through May 29, 2015: American College of Physicians Journal Club, Cochrane Central Register of Controlled Trials, Cochrane Database of Systematic Reviews, Cochrane Methodology Register, Database of Abstracts of Reviews of Effects, Health Technology Assessment, NHS Economic Evaluation Database, Embase, Health and Psycholosocial Instruments, MEDLINE. In addition, the search was limited to English language studies in adult humans only. The search was updated on January 12, 2016, and again on April 28, 2016 to include any recent relevant articles. To ensure a comprehensive search strategy, an academic biomedical librarian was consulted. The search strategy was performed as outlined in Figure 1.

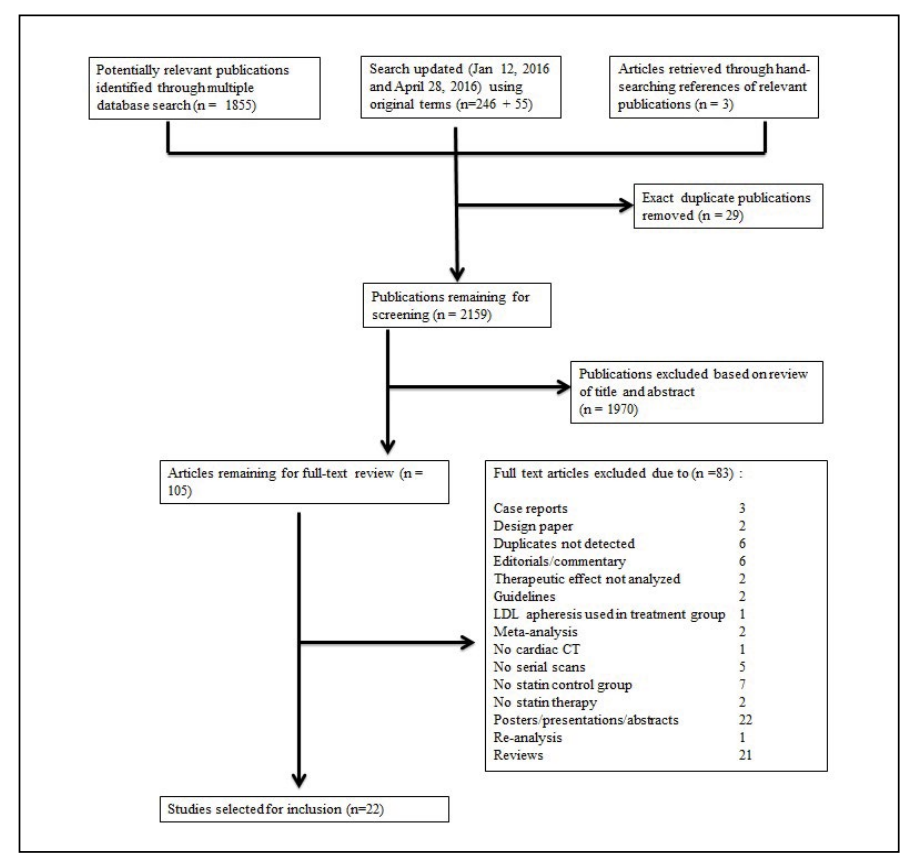

Figure 1) Selection of relevant articles from literature search

${ }^{1}$ University of British Columbia, Department of Medicine, Vancouver, British Columbia, ${ }^{2}$ University of British Columbia, Department of Radiology, Vancouver, British Columbia, ${ }^{3}$ University of Alberta, Mazankowski Heart Institute, Edmonton, Alberta

Correspondence: Dr. G. B. John Mancini, Room 9111, 2775 Laurel Street, Vancouver, BC V5Z 1M9, Columbia, Telephone 604-875-5477, fax 604-875-5471, e-mail mancini@mail.ubc.ca

Received: September 14, 2016, Accepted: December 07, 2016, Published: December 09, 2016

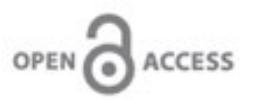

OPEN $\bigcirc$ ACCESS

This open-access article is distributed under the terms of the Creative Commons Attribution Non-Commercial License (CC BY-NC) (http:// creativecommons.org/licenses/by-nc/4.0/), which permits reuse, distribution and reproduction of the article, provided that the original work is properly cited and the reuse is restricted to noncommercial purposes. For commercial reuse, contact reprints@pulsus.com 


\section{Selection criteria}

Inclusion criteria required studies to be original English language peerreviewed publications that quantitatively measured the relationship between documented statin therapy and serial assessments of calcium and non-calciumbased indices of coronary artery disease as measured by CCTA. Case reports, review articles, poster abstracts, conference proceedings, commentaries and guidelines were excluded. In addition, studies with no documented record of statin therapy and studies without a serial measurement were manually excluded. The authors independently selected studies published between 1980 and 2016 as outlined in Figure 1. References from relevant studies were further assessed for additional relevant publications.

\section{Data extraction}

The following data was manually extracted from relevant publications: (1) study design, (2) study population characteristics, (3) number of subjects included in study, (4) specific statin used and comparison treatment, (5) imaging modality, (6) interval between imaging, (7) method used to measure CACS, (8) change in CACS or non-calcium based indices over elapsed time period. Two trained researchers extracted the data in collaboration to ensure that there was agreement between quantitative and qualitative information obtained from each manuscript selected for inclusion.

Individual manuscripts and data extracted were screened for homogeneity of reported outcomes. Due to the significant heterogeneity between reported outcomes, a meta-analysis was not conducted. Instead, a qualitative analysis was used to report our findings.

\section{RESULTS}

\section{Studies identified}

Figure 1 summarizes the screening and selection process. In total, 1858 papers were identified as potentially relevant. Of these, 98 manuscripts were selected for full-text review after initial screening by title and abstract. Finally, 21 studies met the pre-defined criteria after full-text review. Upon updating the search (January 12, 2016), a further 246 papers were screened, of which an additional 7 papers were selected for full-text review, and 1 was selected for inclusion. The search was updated again on April 28, 2016 and 55 additional papers were screened, none of which met the inclusion criteria.

Study characteristics

Of the 22 studies, 9 were randomized controlled trials. The remainder of the studies consisted of retrospective and prospective observational studies. The study populations were highly variable, including HIV-infected patients, patients with chronic kidney disease, and asymptomatic patients undergoing routine screening. The majority of studies used multidetector computed tomography (MDCT) or electron-beam computed tomography (EBCT) as their primary imaging modality to measure CACS. A significant number of studies also included non-calcium based indices measured by CCTA such as plaque volume, non-calcified plaque volume and low attenuation plaque volume. A summary of the studies selected for this review can be found in Table 1, which outlines the study design, study population, inclusion/ exclusion criteria, and imaging modality utilized.

\section{Statin therapy}

Most studies compared some form of statin therapy to no statin therapy or placebo. Three studies compared intensive statin therapy to less intensive therapy. Statin therapy reduced LDL cholesterol in all studies where it was reported. Atorvastatin was the most commonly used statin but effects of Simvastatin, Rosuvastatin, Fluvastatin and Cerivastatin were also reported (Supplementary Table 1).

\section{Coronary artery calcium score progression}

Measures of coronary calcification included Agatston scoring and volumetric calcium scoring. Some studies reported pre-treatment and post-treatment CACS, while others reported only the changeover defined periods. Eighteen studies followed progression of calcium-based indices with statin therapy (Table 2a).

Observational trials included in this review (22-24,32-38) did not consistently demonstrate reduction in progression of CACS. Five studies showed a reduction in CACS with statin therapy $(22-24,33,37)$. Three studies did not demonstrate any significant change. 32,35 Two studies showed increased CACS with statin therapy $(34,38)$. A statistically significant reduction in relative calcium volume score was shown by Mohler et al but this was not reflected in the analysis of Agatston scores which showed no change (36). The remainder of the observational studies assessed non-calcium-based indices of plaque progression.

The randomized controlled trials included in this review $(25-29,31)$ failed to demonstrate any significant reduction in CACS with statin therapy. One study (39) by Lo et al demonstrated reduced non-calcified plaque volume and high-risk plaque features in a small population of HIV-infected individuals with subclinical atherosclerosis, but did not show any significant change

TABLE 1

\section{Study information}

\begin{tabular}{|c|c|c|c|c|c|c|}
\hline $\begin{array}{l}\text { Author and } \\
\text { year }\end{array}$ & Study Design & Study Population & Inclusion & Exclusion & $\begin{array}{l}\text { Imaging } \\
\text { modality }\end{array}$ & Findings \\
\hline $\begin{array}{l}\text { Auscher et al. } \\
\text { (41) } 2015\end{array}$ & $\begin{array}{l}\text { Prospective } \\
\text { randomized } \\
\text { open-label }\end{array}$ & $\begin{array}{l}\text { Documented } \\
\text { STEMI or NSTEMI }\end{array}$ & $\begin{array}{l}\text { Documented STEMI or NSTEMI } \\
\text { according to current guidelines } \\
\text { recruited }<48 \mathrm{~h} \text { after admission }\end{array}$ & $\begin{array}{l}\text { - Ongoing high-dose statin } \\
\text { - } \text { Contraindication to intensive statin therapy } \\
\text { - } \text { Prior or planned CABG } \\
\text { - } \text { Impaired renal function } \\
\text { - }\end{array}$ & MDCT & $\begin{array}{l}\text { Statin therapy } \\
\text { increases dense } \\
\text { calcium volume } \\
\text { but does not } \\
\text { affect total } \\
\text { plaque volume }\end{array}$ \\
\hline $\begin{array}{l}\text { Lo et al. (37) } \\
2015\end{array}$ & $\begin{array}{l}\text { Randomized } \\
\text { double blind } \\
\text { placebo } \\
\text { controlled }\end{array}$ & $\begin{array}{l}\text { HIV-infected } \\
\text { patients with } \\
\text { subclinical } \\
\text { atherosclerosis }\end{array}$ & $\begin{array}{l}\text { - Men and women } 18-60 \text { years of } \\
\text { age with HIV disease } \\
\text { - Stable antiretroviral therapy } \\
\text { - } \text { LDL } 1.81-3.37 \mathrm{mmol} / \mathrm{L} \\
\text { - } \text { Evidence of subclinical } \\
\text { atherosclerosis (plaques on } \\
\text { coronary CTA without clinically } \\
\text { significant stenosis) } \\
\text { - Evidence of arterial inflammation } \\
\text { as assessed by FDG-PET }\end{array}$ & $\begin{array}{l}\text { - Concurrent use of statin } \\
\text { - } \text { Contraindication to statin use } \\
\text { usT or ALT greater than three times the } \\
\text { - Treatment for active liver, renal or } \\
\text { infectious disease } \\
\text { - B-blocker or nitroglycerin use } \\
\text { - Significant radiation exposure with } 1 \text { year } \\
\text { of study } \\
\text { - } \text { Body weight greater than } 136 \mathrm{~kg} \\
\text { - } \text { Pregrgy to iodine contrast } \\
\text { Pregncy or breastfeeding }\end{array}$ & MDCT & $\begin{array}{l}\text { Statins reduce } \\
\text { non-calcified } \\
\text { plaque volume } \\
\text { and high risk } \\
\text { plaque features }\end{array}$ \\
\hline $\begin{array}{l}\text { Lemos et al. } \\
\text { (29) } 2013\end{array}$ & $\begin{array}{l}\text { Open label } \\
\text { randomized } \\
\text { controlled }\end{array}$ & $\begin{array}{l}\text { Nondialyzed CKD } \\
\text { patients }\end{array}$ & $\begin{array}{l}\text { - Older than } 18 \text { years } \\
\text { - Followed by nephrologist by at } \\
\text { least } 3 \text { months }\end{array}$ & $\begin{array}{l}\text { - Presence of chronic inflammatory } \\
\text { diseases } \\
\text { - } \text { Active malignancy } \\
\text { - HIV positive } \\
\text { - } \text { Viral hepatitis } \\
\text { - Chronic steroid use }\end{array}$ & MDCT & $\begin{array}{l}\text { Statin therapy } \\
\text { does not delay } \\
\text { progression of } \\
\text { CAC }\end{array}$ \\
\hline $\begin{array}{l}\text { Zeb et al. (38) } \\
2013\end{array}$ & $\begin{array}{l}\text { Retrospective } \\
\text { observational }\end{array}$ & $\begin{array}{l}\text { Patients being } \\
\text { evaluated for CAD } \\
\text { without known prior } \\
\text { heart disease or } \\
\text { revascularization }\end{array}$ & $\begin{array}{l}\text { - Patients undergoing coronary } \\
\text { CTA between 2006-2009 } \\
\text { - } 2 \text { consecutive scans at least } \\
1 \text { year apart, without prior } \\
\text { known CAD } \\
\text { - Scans with good image } \\
\text { quality }\end{array}$ & $\begin{array}{l}\text { - Scans with significant artifact or poor } \\
\text { image quality } \\
\text { - Interim coronary revascularization }\end{array}$ & MDCT & $\begin{array}{l}\text { Statin therapy } \\
\text { results in } \\
\text { reduced } \\
\text { progression of } \\
\text { low attenuation } \\
\text { plaques and non- } \\
\text { calcified plaques }\end{array}$ \\
\hline
\end{tabular}




\begin{tabular}{|c|c|c|c|c|c|c|}
\hline $\begin{array}{l}\text { Plazak et al. } \\
\text { (28) } 2011\end{array}$ & $\begin{array}{l}\text { Prospective } \\
\text { randomized } \\
\text { double blind } \\
\text { controlled } \\
\text { study }\end{array}$ & $\begin{array}{l}\text { Systemic lupus } \\
\text { erythematosus }\end{array}$ & $\begin{array}{l}\text { At least } 4 \text { of American College } \\
\text { of Rheumatology criteria for SLE } \\
\text { and in stable clinical condition }\end{array}$ & $\begin{array}{l}\text { Patients with known cancer } \\
\text { Clinical symptoms of coronary artery } \\
\text { disease or heart failure, renal failure, } \\
\text { respiratory failure }\end{array}$ & MDCT & $\begin{array}{l}\text { Statin therapy } \\
\text { reduces } \\
\text { progression of } \\
\text { CACS }\end{array}$ \\
\hline $\begin{array}{l}\text { Tenenbaum et } \\
\text { al. (30) } 2011\end{array}$ & Longitudinal & $\begin{array}{l}\text { Patients with stable } \\
\text { angina pectoris }\end{array}$ & & & MDCT & $\begin{array}{l}\text { No change in } \\
\text { CACS with statin } \\
\text { therapy }\end{array}$ \\
\hline $\begin{array}{l}\text { Goh et al. (31) } \\
2010\end{array}$ & $\begin{array}{l}\text { Prospective } \\
\text { longitudinal }\end{array}$ & $\begin{array}{l}\text { Westernized Hong } \\
\text { Kong Chinese } \\
\text { individuals with } \\
\text { chest pain and } \\
\text { coronary risk } \\
\text { factors }\end{array}$ & $\begin{array}{l}\text { Westernized ethnic Chinese } \\
\text { urban inhabitants } \\
\text { - Positive cardiac risk factors and } \\
\text { chest pain } \\
\text { - First presentation chest } \\
\text { symptoms with no prior cardiac } \\
\text { CT }\end{array}$ & $\begin{array}{l}\text { Previous treatment for coronary artery } \\
\text { disease } \\
\text { - Unstable chest symptoms } \\
\text { Patients found to be at high risk requiring } \\
\text { revascularization }\end{array}$ & EBCT & $\begin{array}{l}\text { Statin therapy } \\
\text { causes } \\
\text { regression of } \\
\text { CACS }\end{array}$ \\
\hline $\begin{array}{l}\text { Hoffmann et } \\
\text { al. (39) } 2010\end{array}$ & $\begin{array}{l}\text { Retrospective } \\
\text { longitudinal }\end{array}$ & $\begin{array}{l}\text { Patients who had } \\
\text { repeat MDCT } \\
\text { as follow-up to } \\
\text { initial testing for } \\
\text { suspected CAD }\end{array}$ & $\begin{array}{l}\text { - Consecutive patients undergoing } \\
\text { MDCT as a follow-up to original } \\
\text { CT study } \\
\text { - Referral by primary care } \\
\text { physician }\end{array}$ & & MDCT & $\begin{array}{l}\text { Statin } \\
\text { therapy slows } \\
\text { progression of } \\
\text { non-calcified } \\
\text { plaques }\end{array}$ \\
\hline $\begin{array}{l}\text { Inoue et al. } \\
\text { (40) } 2010\end{array}$ & $\begin{array}{l}\text { Prospective } \\
\text { longitudinal }\end{array}$ & $\begin{array}{l}\text { Patients } \\
\text { undergoing } \\
\text { coronary CTA for } \\
\text { suspected CAD }\end{array}$ & $\begin{array}{l}\text { Patients who underwent } \\
\text { coronary CTA with suspected } \\
\text { coronary artery disease }\end{array}$ & $\begin{array}{l}\text { - Severely calcified lesions on CTA } \\
\text { - Lesion segments with }>75 \% \text { luminal } \\
\text { stenosis } \\
\text { - Prior percutaneous coronary intervention }\end{array}$ & MDCT & $\begin{array}{l}\text { Statin therapy } \\
\text { decreases } \\
\text { plaque and } \\
\text { necrotic core } \\
\text { volumes }\end{array}$ \\
\hline $\begin{array}{l}\text { Anand et al. } \\
\text { (32) } 2007\end{array}$ & $\begin{array}{l}\text { Prospective } \\
\text { longitudinal }\end{array}$ & $\begin{array}{l}\text { Type } 2 \text { diabetics } \\
\text { without prior history } \\
\text { of coronary disease }\end{array}$ & $\begin{array}{l}\text { - Type } 2 \text { diabetes }>1 \text { year } \\
\text { duration } \\
\text { - Age } 30-65 \text { years } \\
\text { No prior history of coronary heart } \\
\text { disease }\end{array}$ & $\begin{array}{l}\text { Typical angina pectoris or angina } \\
\text { equivalent symptoms } \\
\text { - History of positive stress test, myocardial } \\
\text { infarction, heart failure, coronary } \\
\text { revascularization } \\
\text { - Electrocardiographic evidence of Q-wave } \\
\text { myocardial infarction, ischemic ST- or } \\
\text { T-wave changes, complete left bundle } \\
\text { branch block } \\
\text { - History of peripheral vascular disease, } \\
\text { intermittent claudication, stroke or TIA } \\
\text { Renal impairment or severe life } \\
\text { threatening illness }\end{array}$ & EBCT & $\begin{array}{l}\text { Statin use is an } \\
\text { independent } \\
\text { predictor } \\
\text { of CACS } \\
\text { progression }\end{array}$ \\
\hline $\begin{array}{l}\text { Burgstahler et } \\
\text { al. (33) } 2007\end{array}$ & $\begin{array}{l}\text { Prospective } \\
\text { longitudinal }\end{array}$ & $\begin{array}{l}\text { Men with } \\
\text { established } \\
\text { cardiovascular risk, } \\
\text { but no known CAD }\end{array}$ & $\begin{array}{ll}\text { - } & \text { Male patients } \\
\text { - } & \text { Elevated risk of CAD (PROCAM } \\
\text { score }>3^{\text {rd }} \text { quintile) } \\
\text { - Not receiving lipid lowering } \\
\text { therapy }\end{array}$ & & MDCT & $\begin{array}{l}\text { Statin therapy } \\
\text { reduces non- } \\
\text { calcified plaque } \\
\text { burden but does } \\
\text { not affect CACS }\end{array}$ \\
\hline $\begin{array}{l}\text { Mohler et al. } \\
\text { (34) } 2007\end{array}$ & $\begin{array}{l}\text { Single center } \\
\text { prospective } \\
\text { observational } \\
\text { study }\end{array}$ & $\begin{array}{l}\text { Patients with } \\
\text { moderate-severe } \\
\text { aortic stenosis }\end{array}$ & $\begin{array}{l}\text { Aortic valve area } 0.7-2.0 \mathrm{~cm}^{2} \\
\text { EBT and echocardiographic } \\
\text { analysis at baseline and one } \\
\text { year after enrollment }\end{array}$ & & EBCT & $\begin{array}{l}\text { Statin therapy } \\
\text { decreases } \\
\text { progression of } \\
\text { CACS }\end{array}$ \\
\hline $\begin{array}{l}\text { Terry et al. } \\
\text { (25) } 2007\end{array}$ & $\begin{array}{l}\text { Randomized } \\
\text { controlled trial }\end{array}$ & $\begin{array}{l}21 \text { to } 75 \text { years of } \\
\text { age with triglyceride } \\
\text { levels } \\
<600 \mathrm{mg} / \mathrm{dl} \text {. }\end{array}$ & $\begin{array}{ll}\text { - } & \text { Patients } 21-75 \text { years of age } \\
\text { - } & \text { Triglyceride levels }<600 \mathrm{mg} / \mathrm{dl} \\
\text { - } & 1 \text { of the following: } \\
> & \mathrm{HDL} \leq 50 \mathrm{mg} / \mathrm{dl} \\
> & \mathrm{LDL} 100-130 \mathrm{mg} / \mathrm{dl} \\
> & <2 \text { other risk factors that modify } \\
& \text { LDL goal } \\
\text { - } & \mathrm{CAC} \geq 50 \mathrm{U} \text { by Agatston method }\end{array}$ & $\begin{array}{l}\text { Documented history of vascular disease } \\
\text { or diabetes } \\
\text { - Liver aminotransferase levels }>20 \% \text { upper } \\
\text { limit of normal } \\
\text { - Creatinine kinase levels }>50 \% \text { upper limit } \\
\text { of normal } \\
\text { - Creatinine }>1.8 \mathrm{mg} / \mathrm{dl} \\
\text { - Untreated thyroid abnormaities } \\
\text { - Women capable of being pregnant and } \\
\text { not on birth control } \\
\text { - Untreated blood pressure }>140 / 90 \mathrm{mmHg} \\
\text { - Known history or intolerance of } \\
\text { Simvastatin } \\
\text { - Significant incidental findings on baseline } \\
\text { CT } \\
\text { Patients taking other lipid-altering } \\
\text { medications }\end{array}$ & MDCT & $\begin{array}{l}\text { Statin therapy } \\
\text { does not reduce } \\
\text { progression of } \\
\text { CACS }\end{array}$ \\
\hline $\begin{array}{l}\text { Houslay et al. } \\
\text { (24) } 2006\end{array}$ & $\begin{array}{l}\text { Randomized } \\
\text { controlled trial }\end{array}$ & $\begin{array}{l}\text { Patients with } \\
\text { calcific aortic } \\
\text { stenosis and } \\
\text { coronary artery } \\
\text { calcification }\end{array}$ & $\begin{array}{l}\text { Patients aged }>18 \text { years } \\
\text { with calcific aortic stenosis } \\
\text { (grade } 1-3 \text { calcification on } \\
\text { echocardiography } \\
\text { - Peak post-valve velocity of } \geq \\
2.5 \mathrm{~m} / \mathrm{s}\end{array}$ & 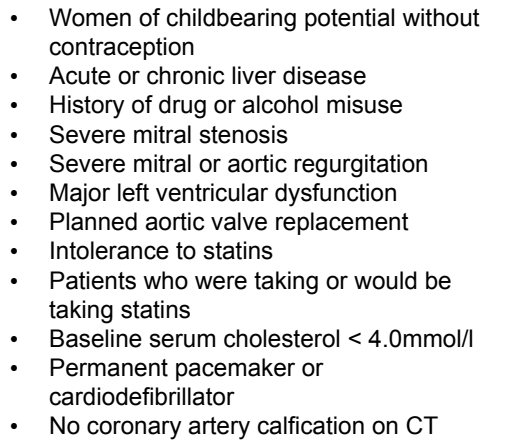 & MDCT & $\begin{array}{l}\text { Statin therapy } \\
\text { does not have } \\
\text { an effect on the } \\
\text { rate of CACS } \\
\text { progression }\end{array}$ \\
\hline
\end{tabular}




\section{Mancini et al}

\begin{tabular}{|c|c|c|c|c|c|c|c|}
\hline $\begin{array}{l}\text { Schmermund } \\
\text { et al. (26) } \\
2006\end{array}$ & $\begin{array}{l}\text { Randomized } \\
\text { controlled trial }\end{array}$ & $\begin{array}{l}\text { Patients with no } \\
\text { history of CAD } \\
\text { and no evidence } \\
\text { of high-grade } \\
\text { coronary stenosis, } \\
\text { and with } \geq 2 \mathrm{CV} \\
\text { risk factors and } \\
C A C \geq 30\end{array}$ & $\begin{array}{l}\text { - Men and women aged } 32-80 \\
\text { years } \\
\text { - Weight less than } 115 \mathrm{~kg} \\
\text { - No history of myocardial } \\
\text { infarction or coronary } \\
\text { revascularization } \\
\text { - No hemodynamically significant } \\
\text { stenosis demonstrated by } \\
\text { angiogram or exercise stress } \\
\text { test } \\
\text { - LDL } 130-250 \mathrm{mg} / \mathrm{dL} \text { without } \\
\text { HMG-CoA reductase inhibitor } \\
\text { therapy, or between } 100-130 \mathrm{mg} / \\
\text { dL with therapy } \\
\text { Triglyceride }<400 \mathrm{mg} / \mathrm{dL} \\
\text { - At least } 2 \text { cardiovascular risk } \\
\text { factors }\end{array}$ & $\begin{array}{l}\dot{\bullet} \\
\dot{ } \\
\text {. } \\
\text {. } \\
\text {. }\end{array}$ & $\begin{array}{l}\text { History of ischemic heart disease } \\
\text { Unstable angina pectoris } \\
\text { Symptomatic chronic heart failure and/or } \\
\text { left ventricular ejection fraction }<40 \% \\
\text { Atrial fibrillation or other arrhythmias that } \\
\text { interfere with ECG-gated triggering of } \\
\text { EBCT } \\
\text { Type } 1 \text { diabetes mellitus or uncontrolled } \\
\text { type } 2 \text { diabetes mellitus } \\
\text { Treatment with bile acid sequestrants, } \\
\text { fibrates, nicotinic acid derivatives, orlistat } \\
\text { Lack of effective contraception or } \\
\text { pregnancy and lactation in women of } \\
\text { childbearing potential }\end{array}$ & EBCT & $\begin{array}{l}\text { Statin therapy } \\
\text { does not } \\
\text { affect CACS } \\
\text { progression }\end{array}$ \\
\hline $\begin{array}{l}\text { Arad et al. } \\
\text { (23) } 2005\end{array}$ & $\begin{array}{l}\text { Randomized } \\
\text { controlled trial }\end{array}$ & $\begin{array}{l}\text { Asymptomatic } \\
\text { apparently healthy } \\
\text { men and women } \\
\text { ages } 50-70 \text { with } \\
\text { CAC scores at } \\
\text { or above } 80^{\text {th }} \\
\text { percentile }\end{array}$ & $\begin{array}{l}\text { Men and women aged } 50-70 \\
\text { years } \\
\text { No history, symptoms or signs } \\
\text { of atherosclerotic cardiovascular } \\
\text { disease }\end{array}$ & $\begin{array}{l}\dot{\bullet} \\
\dot{ } \\
\dot{ } \\
\dot{ } \\
\text { - } \\
\text { - } \\
\text { - }\end{array}$ & $\begin{array}{l}\text { Insulin-dependent diabetes } \\
\text { Triglycerides }>500 \mathrm{mg} / \mathrm{dL} \\
\mathrm{LDL}>175 \mathrm{mg} / \mathrm{dL} \text { in } \mathrm{men} \\
\text { Total cholesterol }>300 \mathrm{mg} / \mathrm{dL} \text { in women } \\
\text { Weight }>136 \mathrm{~kg} \\
\text { Disease likely to cause death within } 5 \\
\text { years } \\
\text { Current therapy with estrogens or } \\
\text { glucocorticoids } \\
\text { Refusal to discontinue lipid-lowering } \\
\text { drugs, vitamin } \mathrm{C} \text { or vitamin } \mathrm{E} \\
\text { Uncontrolled hypertension } \\
\text { LDL }<90 \mathrm{mg} / \mathrm{dL}\end{array}$ & EBCT & $\begin{array}{l}\text { Statin therapy } \\
\text { does not affect } \\
\text { the progression } \\
\text { of CACS }\end{array}$ \\
\hline $\begin{array}{l}\text { Budoff et al. } \\
\text { (35) } 2005\end{array}$ & $\begin{array}{l}\text { Cross- } \\
\text { sectional }\end{array}$ & $\begin{array}{l}\text { Physician-referred } \\
\text { asymptomatic } \\
\text { patients with type } 2 \\
\text { diabetes }\end{array}$ & $\begin{array}{l}\text { - Type } 2 \text { diabetic patients without } \\
\text { evidence of CAD }\end{array}$ & & & EBCT & $\begin{array}{l}\text { Statin therapy } \\
\text { induces a } \\
\text { reduction in the } \\
\text { rate of CACS } \\
\text { progression }\end{array}$ \\
\hline $\begin{array}{l}\text { Raggi et al. } \\
\text { (27) } 2005\end{array}$ & $\begin{array}{l}\text { Randomized } \\
\text { controlled trial }\end{array}$ & $\begin{array}{l}\text { Hyperlipidemic } \\
\text { postmenopausal } \\
\text { women }\end{array}$ & $\begin{array}{l}\text { - Postmenopausal women aged } \\
55-75 \\
\text { - } \\
\text { Menopause as defined by } \\
\text { amenorrhea for at least } 1 \text { year or } \\
\text { receipt of hormone replacement } \\
\text { for at least } 1 \text { year } \\
\text { - } \mathrm{LDL} \geq 130 \mathrm{mg} / \mathrm{dL} \text { for women with } \\
\mathrm{CHD}, \mathrm{CHD} \text { risk equivalents or } \geq \\
2 \text { risk factors and } 10 \text {-year } \mathrm{CHD} \\
\text { risk of } 10-20 \% \\
\text { - } \mathrm{LDL} \geq 160 \mathrm{mg} / \mathrm{dL} \text { for patients with } \\
\geq 2 \mathrm{CHD} \text { risk factors and } 10 \text {-year } \\
\mathrm{CHD} \text { risk of }<10 \% \\
\text { - Patients with } 0-1 \text { risk factors } \\
\text { - Total calcium volume score } \geq 30 \\
\text { at baseline }\end{array}$ & $\begin{array}{l}\dot{ } \\
\dot{ } \\
\dot{ } \\
\dot{ } \\
\dot{ } \\
\dot{ }\end{array}$ & $\begin{array}{l}\text { Patient has contraindication to use of } \\
\text { statins } \\
\text { Treatment with lipid-lowering drugs other } \\
\text { than HRT within } 3 \text { months of screening } \\
\text { Evidence of secondary hyperlipidemia } \\
\text { Renal dysfunction } \\
\text { Uncontrolled Type } 1 \text { or } 2 \text { diabetes mellitus } \\
\text { Myocardial infarction }<6 \text { months before } \\
\text { screening } \\
\text { Uncontrolled hyperthyroidism } \\
\text { Plasma triglyceride }>600 \mathrm{mg} / \mathrm{dL}\end{array}$ & EBCT & $\begin{array}{l}\text { Statin therapy } \\
\text { does not } \\
\text { affect CACS } \\
\text { progression }\end{array}$ \\
\hline $\begin{array}{l}\text { Hecht et al. } \\
\text { (36) } 2003\end{array}$ & Observational & $\begin{array}{l}\text { Asymptomatic } \\
\text { patients with } \\
\text { EBCT evidence } \\
\text { of subclinical } \\
\text { atherosclerosis }\end{array}$ & $\begin{array}{l}\text { Asymptomatic patients who } \\
\text { underwent serial EBCT at } \\
\text { intervals of }>1 \text { year }\end{array}$ & & & EBCT & $\begin{array}{l}\text { Statin therapy } \\
\text { increases CACS }\end{array}$ \\
\hline $\begin{array}{l}\text { Achenbach et } \\
\text { al. (22) } 2002\end{array}$ & $\begin{array}{l}\text { Prospective } \\
\text { cohort }\end{array}$ & $\begin{array}{l}\text { Patients who } \\
\text { underwent EBCT, } \\
\text { CAC score } \geq 20 \text {, no } \\
\text { known CAD, LDL } \\
>130 \mathrm{mg} / \mathrm{dL}\end{array}$ & $\begin{array}{ll}\text { - } & \text { Coronary calcification in EBT } \\
\text { - } & \text { LDL }>130 \mathrm{mg} / \mathrm{dL} \\
\text { - } & \text { No lipid-lowering therapy } \\
\text { - } & \text { Time interval of at least } 12 \\
\text { months since EBT scan with } \\
\text { documented Agatston score } \geq 20 \\
\text { - } \text { No known CAD or symptoms } \\
\text { suggestive of disease } \\
\text { - } \text { Sinus rhythm } \\
\text { - Normal renal function }\end{array}$ & & & EBCT & $\begin{array}{l}\text { Statin therapy } \\
\text { reduces } \\
\text { progression of } \\
\text { CACS }\end{array}$ \\
\hline $\begin{array}{l}\text { Budoff et al. } \\
\text { (21) } 2000\end{array}$ & Observational & $\begin{array}{l}\text { Asymptomatic } \\
\text { patients referred by } \\
\text { primary physician } \\
\text { to evaluate the } \\
\text { presence and } \\
\text { amount of coronary } \\
\text { calcium }\end{array}$ & $\begin{array}{l}\text { Asymptomatic patients who } \\
\text { underwent } 2 \text { consecutive EBT } \\
\text { scans at least } 12 \text { months apart }\end{array}$ & $\cdot$ & $\begin{array}{l}\text { Documented CAD before entry into the } \\
\text { study } \\
\text { Inadequate images for analysis on either } \\
\text { EBT scan }\end{array}$ & EBCT & $\begin{array}{l}\text { Statin therapy } \\
\text { induces a } \\
\text { reduction in the } \\
\text { rate of CACS } \\
\text { progression }\end{array}$ \\
\hline $\begin{array}{l}\text { Callister et al. } \\
\text { (20) } 1998\end{array}$ & $\begin{array}{l}\text { Retrospective } \\
\text { observational }\end{array}$ & $\begin{array}{l}\text { Patients with no } \\
\text { history of CAD, } \\
\text { referred by primary } \\
\text { care physician for } \\
\text { screening EBCT }\end{array}$ & $\begin{array}{l}\text { - Asymptomatic patients with no } \\
\text { history of CAD } \\
\text { - Referred by primary care } \\
\text { physicians for serial EBCT at } \\
\text { intervals of } 12-15 \text { months }\end{array}$ & $\dot{\bullet}$ & $\begin{array}{l}\text { Inadequate image quality } \\
\text { Initial calcium volume score }<30\end{array}$ & EBCT & $\begin{array}{l}\text { Statin therapy } \\
\text { reduces CACS } \\
\text { progression }\end{array}$ \\
\hline
\end{tabular}

in CACS. Another study by Plazak et al (30) showed a reduction in CACS after 1 year of statin therapy in a group of patients with systemic lupus erythematosus (SLE).
Progression of non-calcium-based indices

Several observational studies have reported changes in plaque volume as derived from CCTA (Table 2b) (35,40-42). 
TABLE 2a

Calcium-based indices

\begin{tabular}{|c|c|c|c|c|c|}
\hline Study & $\begin{array}{l}\text { Length of } \\
\text { Treatment }\end{array}$ & Calcium Measurement Method & $\begin{array}{l}\text { CAC Progression in Untreated } \\
\text { Group }\end{array}$ & $\begin{array}{l}\text { CAC Progression in Treated } \\
\text { Group }\end{array}$ & Significant difference \\
\hline Lo et al. 2015 & 1 year & $\begin{array}{l}\text { Agatston score, calcium mass, } \\
\text { calcium volume, calcium density }\end{array}$ & $\begin{array}{l}\text { Agatston Score } \\
\Delta 1.7\end{array}$ & $\begin{array}{l}\text { Agatston Score } \\
\Delta 0.9\end{array}$ & $\begin{array}{l}\text { Agatston Score } \\
p=0.74\end{array}$ \\
\hline Lemos et al. 2013 & 24 months & Agatston score & $\begin{array}{l}\text { Agatston Score } \\
\Delta 99.7+/-190.8 \text { (absolute) } \\
\Delta 56.5+/-70.8 \% \text { (relative) }\end{array}$ & $\begin{array}{l}\text { Agatston Score } \\
\Delta 99.3+/-283.7 \text { (absolute) } \\
\Delta 69.6+/-74.3 \% \text { (relative) }\end{array}$ & $\begin{array}{l}\text { Agatston Score } \\
p=0.28 \text { (absolute) } \\
p=0.35 \text { (relative) }\end{array}$ \\
\hline Plazak et al. 2011 & 1 year & Agatston score & $\begin{array}{l}\text { Agatston Score } \\
32.1+/-39.1 \text { vs. } 59.5+/-54.4\end{array}$ & $\begin{array}{l}\text { Agatston Score } \\
44.8+/-50.6 \text { vs. } 54.9+/-62.5\end{array}$ & $\begin{array}{l}\text { Agatston Score } \\
p<0.05 \text { (untreated) } \\
p=N S \text { (treated) }\end{array}$ \\
\hline Tenenbaum et al. 2011 & Median 5.6 years & Coronary calcification score & $\begin{array}{l}\text { Total calcium score } \\
\Delta 452+/-515\end{array}$ & $\begin{array}{l}\text { Total calcium score } \\
\Delta 495+/-588\end{array}$ & $\begin{array}{l}\text { Total calcium score } \\
p=0.512\end{array}$ \\
\hline Goh et al. 2010 & $10+/-1.5$ years & Agatston score & $\begin{array}{l}\text { Agatston Score } \\
\Delta 33.2 \% / \text { year }\end{array}$ & $\begin{array}{l}\text { Agatston Score } \\
\Delta 24 \% / \text { year }\end{array}$ & $\begin{array}{l}\text { Agatston Score } \\
p<0.001\end{array}$ \\
\hline Anand et al. 2007 & $\begin{array}{l}\text { Mean follow-up } \\
2.5+/-0.4 \text { years }\end{array}$ & $\begin{array}{l}\text { Agatston and volumetric calcium } \\
\text { scores }\end{array}$ & $\begin{array}{l}\text { CACS } \\
\Delta 6 \mathrm{~mm}^{3} / \text { year }\end{array}$ & $\begin{array}{l}\text { CACS } \\
\Delta 25 \mathrm{~mm}^{3} / \text { year }\end{array}$ & $\begin{array}{l}\text { Statin use as an } \\
\text { independent predictor } \\
\text { of CAC progression } \\
\text { (OR2.27, } p=0.001)\end{array}$ \\
\hline Burgstahler et al. 2007 & $488+/-138$ days & $\begin{array}{l}\text { Agatston score, noncalcified } \\
\text { plaques and volumetric plaque } \\
\text { burden }\end{array}$ & $\begin{array}{l}\text { Agatston Score } \\
873+/-1011 \text { vs. } 1017+/-1268 \\
\Delta+32 \%\end{array}$ & $\begin{array}{l}\text { Agatston Score } \\
261+/-301 \text { vs. } 293+/-366 \\
\Delta+17 \%\end{array}$ & $\begin{array}{l}\text { Agatston Score } \\
p>0.05 \text { (untreated) } \\
p=0.59 \text { (treated) }\end{array}$ \\
\hline Mohler et al. 2007 & 1 year & $\begin{array}{l}\text { Coronary artery calcium volume } \\
\text { Agatston score }\end{array}$ & $\begin{array}{l}\text { Calcium volume score } \\
\Delta 19.2+/-308.9 \text { (absolute) } \\
\Delta 58.2+/-76.3 \% \text { (relative) } \\
\text { Agatston Score } \\
\Delta-45.2+/-416 \text { (absolute) } \\
\Delta 15.8+/-40.9 \% \text { (relative) }\end{array}$ & $\begin{array}{l}\text { Calcium volume score } \\
\Delta 59.0+/-435.3 \text { (absolute) } \\
\Delta 16.9+/-52.7 \% \text { (relative) } \\
\text { Agatston Score } \\
\Delta 38.6+/-524.7 \text { (absolute) } \\
\Delta 14.8+/-53.8 \% \text { (relative) }\end{array}$ & $\begin{array}{l}\text { Calcium volume score } \\
p=0.56 \text { (absolute) } \\
p=0.02 \text { (relative) } \\
\text { Agatston Score } \\
p=0.92 \text { (absolute) } \\
p=0.71 \text { (relative) }\end{array}$ \\
\hline Terry et al. 2007 & $\begin{array}{l}6 \text { months and } 12 \\
\text { months }\end{array}$ & CAC Agatston core & $\begin{array}{l}\text { Agatston Score } \\
659+/-116 \text { vs. } 691+/-24 \\
\Delta+5 \%\end{array}$ & $\begin{array}{l}\text { Agatston Score } \\
593+/-132 \text { vs. } 645+/-24 \\
\Delta+9 \%\end{array}$ & $\begin{array}{l}\text { Agatston Score } \\
p=0.12\end{array}$ \\
\hline Houslay et al. 2006 & Median 2 years & Agatston Score & $\begin{array}{l}\text { Agatston Score } \\
\Delta 18 \% / \text { year }\end{array}$ & $\begin{array}{l}\text { Agatston Score } \\
\Delta 26 \% / \text { year }\end{array}$ & $\begin{array}{l}\text { Agatston Score } \\
p=0.18\end{array}$ \\
\hline $\begin{array}{l}\text { Schmermund et al. } \\
2006\end{array}$ & 12 months & $\begin{array}{l}\text { Agatston CAC score and } \\
\text { calcium volume score }\end{array}$ & $\begin{array}{l}\text { Atorvastatin } 10 \mathrm{mg} \\
\text { Agatston Score } \\
\triangle 26 \% \\
\text { CAC Volume Score } \\
\Delta 25 \%\end{array}$ & $\begin{array}{l}\text { Atorvastatin } 80 \mathrm{mg} \\
\text { Agatston Score } \\
\Delta 28 \% \\
\text { CAC Volume Score } \\
\Delta 27 \%\end{array}$ & $\begin{array}{l}\text { Agatston Score } \\
p=0.7 \\
\text { CAC Volume Score } \\
p=0.6\end{array}$ \\
\hline Arad et al. 2005 & $\begin{array}{l}\text { Mean follow-up } \\
4.3 \text { years }\end{array}$ & Agatston score & $\begin{array}{l}\text { Agatston Score } \\
\Delta 323+/-385 \text { (absolute) } \\
\Delta 73+/-93 \% \text { (relative) }\end{array}$ & $\begin{array}{l}\text { Agatston Score } \\
\triangle 331+/-421 \text { (absolute) } \\
\Delta 81+/-89 \% \text { (relative) }\end{array}$ & $\begin{array}{l}\text { Agatston Score } \\
p=0.80 \text { (absolute) } \\
p=0.76 \text { (relative) }\end{array}$ \\
\hline Budoff et al. 2005 & $27+/-15$ months & Agatston score & $\begin{array}{l}\text { CAC Progression } \\
32 \% / \text { year }\end{array}$ & $\begin{array}{l}\text { CAC Progression } \\
18 \% / \text { year }\end{array}$ & $\begin{array}{l}\text { CAC Progression } \\
p=0.02\end{array}$ \\
\hline Raggi et al. 2005 & 12 months & Calcium volume score & $\begin{array}{l}\text { Pravastatin } 40 \mathrm{mg} \\
\text { Calcium Volume Score } \\
\Delta 30.9 \text { (absolute) } \\
\Delta 19.8 \% \text { (relative) }\end{array}$ & $\begin{array}{l}\text { Atorvastatin } 80 \mathrm{mg} \\
\text { Calcium Volume Score } \\
\Delta 28.5 \text { (absolute) } \\
\Delta 20.1 \% \text { (relative) }\end{array}$ & $\begin{array}{l}\text { Calcium Volume Score } \\
p=0.21 \text { (absolute) } \\
p=0.64 \text { (relative) }\end{array}$ \\
\hline Hecht et al. 2003 & $\begin{array}{l}1.2+/-0.7 \text { years } \\
\text { for treated } \\
1.4+/-0.5 \text { years } \\
\text { for untreated }\end{array}$ & $\begin{array}{l}\text { Coronary calcium and calcium } \\
\text { volume scores }\end{array}$ & $\begin{array}{l}\text { Calcium Score } \\
\Delta 28+/-44 \text { (absolute) } \\
\Delta 10.4 \% / \text { year (relative) } \\
\text { Volume Score } \\
\Delta 22+/-39 \text { (absolute) } \\
\Delta 10.7 \% \text { /year (relative) }\end{array}$ & $\begin{array}{l}\text { Calcium Score } \\
\Delta 41+/-145 \text { (absolute) } \\
\Delta 8.9 \% / \text { year (relative) } \\
\text { Volume Score } \\
\Delta 35+/-91 \text { (absolute) } \\
\Delta 9.6 \% / \text { year (relative) }\end{array}$ & $\begin{array}{l}\text { Calcium Score } \\
p<0.001 \text { (absolute) } \\
\text { Volume Score } \\
p<0.001 \text { (absolute) }\end{array}$ \\
\hline Achenbach et al. 2002 & $\begin{array}{l}\text { EBCT performed } \\
\text { on patients with } \\
\text { mean interval } \\
\text { of } 14 \text { months } \\
\text { without treatment, } \\
\text { then again after } \\
12 \text { months of } \\
\text { treatment }\end{array}$ & $\begin{array}{l}\text { Volumetric calcium score, } \\
\text { Agatston score }\end{array}$ & $\begin{array}{l}\text { Agatston Score } \\
\Delta 28 \text { (absolute) } \\
\Delta 25 \% \text { (relative) } \\
\text { Volume Score } \\
\Delta 25 \mathrm{~mm}^{3} \text { (absolute) } \\
\Delta 25 \% \text { (relative) }\end{array}$ & $\begin{array}{l}\text { Agatston Score } \\
\Delta 20 \text { (absolute) } \\
\Delta 11 \% \text { (relative) } \\
\text { Volume Score } \\
\Delta 11 \mathrm{~mm}^{3} \text { (absolute) } \\
\Delta 8.8 \% \text { (relative) }\end{array}$ & $\begin{array}{l}\text { Agatston Score } \\
p=0.07 \text { (absolute) } \\
p=0.002 \text { (relative) } \\
\text { Volume Score } \\
p=0.01 \text { (absolute) } \\
p=0.0001 \text { (relative) }\end{array}$ \\
\hline Budoff et al. 2000 & $2.2+/-1.1$ years & Agatston score & $\begin{array}{l}\text { Agatston Score } \\
\Delta 39+/-12 \% / \text { year }\end{array}$ & $\begin{array}{l}\text { Agatston Score } \\
\Delta 15+/-8 \% / \text { year }\end{array}$ & $\begin{array}{l}\text { Agatston Score } \\
p<0.001\end{array}$ \\
\hline Callister et al. 1998 & $\begin{array}{l}13.7+/-0.6 \\
\text { months }\end{array}$ & Volumetric calcium score & $\begin{array}{l}\text { Calcium volume score } \\
\Delta 52+/-36 \%\end{array}$ & $\begin{array}{l}\text { Calcium volume score } \\
\Delta 5+/-28 \%\end{array}$ & $\begin{array}{l}\text { Calcium volume score } \\
p<0.001\end{array}$ \\
\hline
\end{tabular}

TABLE 2b

Non-calcium-based indices

\begin{tabular}{|c|c|c|c|c|c|}
\hline Study & $\begin{array}{l}\text { Length of } \\
\text { Treatment }\end{array}$ & $\begin{array}{l}\text { Calcium Measurement } \\
\text { Method }\end{array}$ & $\begin{array}{l}\text { CAC Progression in Untreated } \\
\text { Group }\end{array}$ & $\begin{array}{l}\text { CAC Progression in Treated } \\
\text { Group }\end{array}$ & Significant difference \\
\hline $\begin{array}{l}\text { Auscher et al. } \\
2015\end{array}$ & 12 months & $\begin{array}{l}\text { Plaque volume, plaque } \\
\text { composition, total dense } \\
\text { calcium volume }\end{array}$ & $\begin{array}{l}\text { Total plaque volume } \\
2084.7+/-613.2 \mathrm{~mm}^{3} \mathrm{vs} .2103 .7+/- \\
628.8 \mathrm{~mm}^{3} \\
\Delta 19.1+/-190.2 \mathrm{~mm}^{3} \\
\text { Dense calcium volume (median) } \\
24.1[9 ; 81] \mathrm{mm}^{3} \mathrm{vs} .21 .5[12 ; 79] \mathrm{mm}^{3} \\
\Delta 1.9[-6 ; 8] \mathrm{mm}^{3}\end{array}$ & $\begin{array}{l}\text { Total plaque volume } \\
2134.5+/-569.6 \mathrm{~mm}^{3} \mathrm{vs} .2177 .5 \\
+/-566.9 \mathrm{~mm}^{3} \\
\Delta 43.5+/-225.8 \mathrm{~mm}^{3} \\
\text { Dense calcium volume (median) } \\
37.0[12 ; 71] \mathrm{mm}^{3} \mathrm{vs} .45 .0 \text { [ } 17 ; \\
82] \mathrm{mm}^{3} \\
\Delta 10.6[-0.13 ; 21.4] \mathrm{mm}^{3}\end{array}$ & $\begin{array}{l}\text { Total plaque volume } \\
p=0.57 \\
\text { Dense calcium volume } \\
p=<0.001\end{array}$ \\
\hline
\end{tabular}




\section{Mancini et al}

\begin{tabular}{|c|c|c|c|c|c|}
\hline Lo et al. 2015 & 1 year & $\begin{array}{l}\text { Agatston score, calcium } \\
\text { mass, calcium volume, } \\
\text { calcium density }\end{array}$ & $\begin{array}{l}\text { Non-calcified plaque volume } \\
\Delta 6.7 \mathrm{~mm}^{3} \\
\text { Total plaque volume } \\
\Delta 12.0 \mathrm{~mm}^{3}\end{array}$ & $\begin{array}{l}\text { Non-calcified plaque volume } \\
\Delta-8.2 \mathrm{~mm}^{3} \\
\text { Total plaque volume } \\
\Delta-0.8 \mathrm{~mm}^{3}\end{array}$ & $\begin{array}{l}\text { Non-calcified plaque } \\
\text { volume } \\
p=0.03 \\
\text { Total plaque volume } \\
p=0.02\end{array}$ \\
\hline Zeb et al. 2013 & $\begin{array}{l}\text { Mean follow-up } 406 \\
+/-92 \text { days }\end{array}$ & $\begin{array}{l}\text { Volumetric assessment of } \\
\text { low attenuation plaque, } \\
\text { non-calcified and calcified } \\
\text { plaque }\end{array}$ & $\begin{array}{l}\text { Low attenuation plaque } \\
38.1+/-55.8 \mathrm{~mm}^{3} \text { vs } 45.3+/-60.4 \mathrm{~mm}^{3} \\
\Delta 5.9+/-23.1 \mathrm{~mm}^{3} \\
\text { Non-calcified } \\
137.7+/-172.8 \mathrm{~mm}^{3} \text { vs. } 150.1+/-184.9 \\
\mathrm{~mm}^{3} \\
\Delta 13.8+/-76.6 \mathrm{~mm}^{3} \\
\text { Calcified } \\
262.5+/-375.5 \mathrm{~mm}^{3} \text { vs. } 274.1+/-375.9 \\
\mathrm{~mm}^{3} \\
\Delta 10.0+/-53.2 \mathrm{~mm}^{3}\end{array}$ & $\begin{array}{l}\text { Low attenuation plaque } \\
44.9+/-50.2 \mathrm{~mm}^{3} \text { vs } 28.6+/-35.6 \\
\mathrm{~mm}^{3} \\
\Delta-12.2+/-19.2 \mathrm{~mm}^{3} \\
\text { Non-calcified } \\
166.0+/-162.3 \mathrm{~mm}^{3} \text { vs. } 114.4+/- \\
135.3 \mathrm{~mm}^{3} \\
\Delta-47.7+/-71.9 \mathrm{~mm}^{3} \\
\text { Calcified } \\
292.7+/-304 \mathrm{~mm}^{3} \text { vs. } 326.4+/- \\
339 \mathrm{~mm}^{3} \\
\Delta 29.3+/-67.9 \mathrm{~mm}^{3}\end{array}$ & $\begin{array}{l}\text { Low attenuation plaque } \\
p=<0.001 \\
\text { Non-calcified } \\
p=<0.001 \\
\text { Calcified } \\
p=0.245\end{array}$ \\
\hline $\begin{array}{l}\text { Hoffmann et al. } \\
2010\end{array}$ & $25+/-3$ months & $\begin{array}{l}\text { Volumetric plaque } \\
\text { assessment }\end{array}$ & \multicolumn{2}{|c|}{$\begin{array}{l}\text { Linear random intercept model showed growth rate of non-calcified } \\
\text { plaques were significantly slowed by statin }(P=0.01, B 2=-0.0036)\end{array}$} & $p=0.01$ \\
\hline $\begin{array}{l}\text { Inoue et al. } \\
2010\end{array}$ & Median 12 months & $\begin{array}{l}\text { Total plaque volume, } \\
\text { low attenuation plaque } \\
\text { volume, lumen volume, } \\
\text { remodeling index }\end{array}$ & $\begin{array}{l}\text { Total plaque volume } \\
94.4+/-21.2 \mathrm{~mm}^{3} \text { vs. } 98.4+/-28.6 \\
\mathrm{~mm} 3 \\
\text { Low attenuation plaque } \\
2.1+/-3.0 \mathrm{~mm}^{3} \text { vs. } 2.3+/-3.6 \mathrm{~mm}^{3}\end{array}$ & $\begin{array}{l}\text { Total plaque volume } \\
92.3+/-37.7 \mathrm{~mm}^{3} \mathrm{vs} .76 .4+/- \\
26.5 \mathrm{~mm} 3 \\
\text { Low attenuation plaque } \\
\begin{array}{l}4.9+/-7.8 \mathrm{~mm}^{3} \text { vs. } 1.3+/-2.3 \\
\mathrm{~mm}^{3}\end{array}\end{array}$ & $\begin{array}{l}\text { Total plaque volume } \\
p=0.48 \text { (untreated) } \\
p<0.01 \text { (treated) } \\
\text { Low attenuation plaque } \\
p=0.91 \text { (untreated) } \\
p=0.01 \text { (treated) }\end{array}$ \\
\hline $\begin{array}{l}\text { Burgstahler et } \\
\text { al. } 2007\end{array}$ & $488+/-138$ days & $\begin{array}{l}\text { Agatston score, } \\
\text { noncalcified plaques and } \\
\text { volumetric plaque burden }\end{array}$ & $\begin{array}{l}\text { Total plaque burden } \\
0.647+/-0.607 \mathrm{~mL} \text { vs. } 0.628+/-0.523 \\
\mathrm{~mL} \\
\text { Noncalcified plaques } \\
\text { "Enlargement of plaque volume" }\end{array}$ & $\begin{array}{l}\text { Total plaque burden } \\
0.149+/-0.108 \mathrm{~mL} \text { vs. } 0.128+/- \\
0.075 \mathrm{~mL} \\
\text { Noncalcified plaques } \\
0.042+/-0.029 \mathrm{~mL} \text { vs. } 0.030+/- \\
0.014 \mathrm{~mL}\end{array}$ & $\begin{array}{l}\text { Total plaque burden } \\
p=0.228 \text { (treated) } \\
p=0.81 \text { (untreated) } \\
\text { Noncalcified plaques } \\
p<0.05 \text { (treated) }\end{array}$ \\
\hline
\end{tabular}

Three of these studies demonstrated a reduction in the progression of non-calcified plaque volume associated with statin therapy $(35,40,41)$. In addition, Inoue et al showed that statin therapy results in a reduction of both plaque volume and necrotic core volume, implying improved plaque stability (42).

Recent randomized controlled trials have included assessments of coronary plaque from CCTA measurements, including plaque volume, composition and vulnerability $(39,43)$. Auscher et al showed that early aggressive lipid lowering therapy increases dense calcium volume, but did not significantly affect plaque volume in patients with acute myocardial infarction (43). Lo et al demonstrated a reduction in non-calcified plaque volume and other high-risk plaque features in a small group of HIV-infected patients treated with statins (39).

\section{DISCUSSION}

This review identified 22 articles that studied the relationship between statin therapy, CACS and non-calcified plaque changes. The data were not amenable to meta-analysis due to the heterogeneity of reported variables between studies. While multiple observational studies suggested that statin therapy may reduce CACS, this was not confirmed in prospective randomized controlled trials, the one exception being a small randomized trial suggesting that statin therapy may reduce CACS in a small group of SLE patients.

Thus, there is no firm evidence that statin therapy reduces progression of CACS. In fact, a recent meta-analysis of 2 randomized trials suggested that high-dose and long-term statin therapy increases CACS (44). Thus CACS change is an inadequate mean to assess atherosclerotic plaque progression in the setting of statin therapy.

In recent studies using CCTA, investigators have assessed various plaque features using non-calcium based indices such as plaque volume, non-calcified plaque volume and low attenuation plaque volume (Table $2 \mathrm{~b})$. Although there is suggestive evidence that total plaque volume may be improved by statin therapy, there is only one prospective RCT that has shown this in a selective population of HIV-infected individuals (39). Whether this change is associated with a reduction in cardiovascular events is

\section{REFERENCES}

1. Greenland P, Labree L, Azen SP, et al. Coronary artery calcium score combined with framingham score for risk prediction in asymptomatic Individuals. JAMA 2004;291:210-15.

2. Kondos GT, Hoff JA, Sevrukov A, et al. Electron-beam tomography coronary artery calcium and cardiac events: A 37-month follow-up of 5635 initially awaiting demonstration. Further randomized controlled studies are required to determine whether serial CCTA measurements of non-calcium based indices are affected by statin therapy.

Treatment length must be considered when interpreting the results of these articles. Most of the studies selected included study lengths of 1 2 years. It is not clear whether the true effect of statin therapy on CACS can be accurately interpreted over this short time period. Studies using intravascular ultrasound (IVUS) have been performed over similar periods and have demonstrated significant regression of coronary atherosclerosis but increases in dense calcium composition, a result similar to what we note with the studies included in this systematic review $(18,19,45-47)$.

\section{CONCLUSION}

In conclusion, while statin therapy has been shown to affect plaque progression in studies using alternate imaging modalities, this effect has not been convincingly replicated in trials using calcium-based indices. Thus, CACS is not suitable for monitoring the effectiveness of statin therapy on atherosclerosis. Recent CCTA studies suggest that other features of coronary plaque progression such as non-calcified plaque and low attenuation plaque may be favourably affected by statin therapy. As our understanding of coronary plaque progression continues to evolve, future prospective trials are necessary to determine if progression of non-calcium based plaque indices are associated with favourable or unfavourable outcomes.

\section{ACKNOWLEDGEMENT}

We would like to acknowledge Dean Giustini, University of British Columbia Library, for his contributions and input regarding search strategies and optimization.

\section{DISCLOSURES}

Wan, Tashakkor and Mancini: None

Leipsic: Circle Cardiovascular Imaging, GE, Samsung, Phillips Raggi: None

asymptomatic low- to intermediate-risk adults. Circulation 2003;107:2571-76

3. O’Malley PG, Taylor AJ, Jackson JL, et al. Prognostic Value of coronary electron- beam computed tomography for coronary heart disease events in asymptomatic populations. Am J Cardiol 2000;85:945-948.

4. Pletcher MJ, Tice JA, Pignone M, et al. Using the coronary artery calcium score to predict coronary heart disease events. Arch Intern Med 2004; $164: 1285-92$ 
5. Shaw LJ, Raggi P, Schisterman E, et al. Prognostic value of cardiac risk factors and coronary artery calcium screening for all-cause mortality. Radiology 2003;228:826-33.

6. Elias-Smale SE, Proença RV, Koller MT, et al. Coronary calcium score improves classification of coronary heart disease risk in the elderly: The Rotterdam study. J Am Coll Cardiol 2010;56:1407-14.

7. Erbel R, Möhlenkamp S, Moebus S, et al. Coronary risk stratification, discrimination, and reclassification improvement based on quantification of subclinical coronary atherosclerosis: The Heinz Nixdorf Recall study. J Am Coll Cardiol 2010;56:1397-1406.

8. Polonsky TS, Mcclelland RL, Jorgensen NW, et al. Coronary Artery calcium score and risk classification for coronary heart disease prediction. JAMA 2010;303:1610-16.

9. Greenland P, Alpert JS, Beller GA, et al. 2010 ACCF/AHA guideline for assessment of cardiovascular risk in asymptomatic adults: a report of the American College of Cardiology Foundation/American Heart Association Task Force on Practice Guidelines. J Am Coll Cardiol 2010;56:e50-e103.

10. Heart Protection Study Collaborative Group. MRC/BHF Heart Protection Study of cholesterol lowering with simvastatin in 20536 high-risk individuals : a randomised placebo- controlled trial. Lancet 2002;360:7-22.

11. LaRosa JC, Grundy SM, Waters DD, et al. Intensive lipid lowering with atorvastatin in patients with stable coronary disease. NEJM 2005;352:1425-35.

12. The long-term intervention with pravastatin in ischaemic disease study group. Prevention of cardiovascular events and death with pravastatin in patients with coronary heart disease and a broad range of initial cholesterol levels. NEJM 1998;339:1349-57.

13. Sacks FM, Pfeffer MA, Moye LA, et al. The effect of pravastatin on coronary events after myocardial infarction in patients with average cholesterol levels. NEJM 1996;335:1001-09.

14. Scandanavian Simvastatin Survival Study Group. Randomised trial of cholesterol lowering in 4444 patients with coronary heart disease: The Scandinavian Simvastatin Survival Study (4S). Lancet 1994;344:1383-89.

15. Shepherd J, Cobbe SM, Ford I, et al. Prevention of coronary heart disease with pravastatin in men with hypercholesterolemia. NEJM 1995;333:1301-07.

16. Downs JR, Clearfield M, Weis S, et al. Primary prevention of acute coronary events with lovastatin in men and women with average cholesterol levels. JAMA 1998;279:1615-22

17. Pedersen TR, Faergeman O, Kastelein JJP, et al. High-Dose atorvastatin vs usual-dose simvastatin for secondary prevention after myocardial infarction. JAMA 2005;294:2437-46

18. Nissen SE, Nicholls SJ, Siphani I, et al. Effect of very high-intensity statin therapy on regression of coronary atherosclerosis. JAMA 2006;295:1556-65.

19. Nicholls SJ, Ballantyne CM, Barter PJ, et al. Effect of two intensive statin regimens on progression of coronary disease. NEJM 2011;365:2078-87.

20. Puri R, Nicholls SJ, Shao M, et al. Impact of statins on serial coronary calcification during atheroma progression and regression. J Am Coll Cardiol 2015;65:1273-82

21. Yla-Herttuala S, Bentzon JF, Daemen M, et al. Stabilization of atherosclerotic plaques: an update. Eur Heart J 2013;34:3251-58.

22. Callister TQ, Raggi P, Cooil B, et al. Effect of HMG-CoA reductase inhibitors on coronary artery disease as assessed by electron-beam computed tomography. NEJM 1998;339:1972-78.

23. Budoff MJ, Lane KL, Bakhsheshi $\mathrm{H}$, et al. Rates of progression of coronary calcium by electron beam tomography. Am J Cardiol 2000;86:8-11.

24. Achenbach S, Ropers D, Pohle K, et al. Influence of lipid-lowering therapy on the progression of coronary artery calcification: a prospective evaluation. Circulation 2002;106:1077-82.

25. Arad Y, Spadaro LA, Roth M, et al. Treatment of asymptomatic adults with elevated coronary calcium scores with atorvastatin, vitamin c, and vitamin $\mathrm{E}$. J Am Coll Cardiol 2005;46:166-72.

26. Houslay ES, Cowell SJ, Prescott RJ, et al. Progressive coronary calcification despite intensive lipid-lowering treatment: a randomised controlled trial. Heart 2006;92:1207-12.

27. Terry JG, Carr JJ, Kouba EO, et al. Effect of simvastatin $(80 \mathrm{mg})$ on coronary and abdominal aortic arterial calcium (from the coronary artery calcification treatment with zocor [CATZ] study). Am J Cardiol 2007;99:1714-17.
28. Schmermund A, Achenbach S, Budde T, et al. Effect of intensive versus standard lipid-lowering treatment with atorvastatin on the progression of calcified coronary atherosclerosis over 12 months: A Multicenter, Randomized, Double-Blind Trial. Circulation 2006;113:427-37.

29. Raggi P, Davidson M, Callister TQ, et al. Aggressive versus moderate lipid-lowering therapy in hypercholesterolemic postmenopausal women: beyond endorsed lipid lowering with EBT scanning (BELLES). Circulation 2005; 112:563-71.

30. Plazak W, Gryga K, Dziedzic H, et al. Influence of atorvastatin on coronary calcifications and myocardial perfusion defects in systemic lupus erythematosus patients: a prospective, randomized, double-masked, placebocontrolled study. Arthritis Res Ther 2011;13:R117.

31. Lemos MM, Watanabe R, Carvalho AB, et al. Effect of rosuvastatin and sevelamer on the progression of coronary artery calcification in chronic kidney disease: a pilot study. Clin Nephrol 2013;80:1-8.

32. Tenenbaum A, Shemesh J, Koren-Morag N, et al. Long-term changes in serum cholesterol level does not influence the progression of coronary calcification. Int J Cardiol 2011;150:130-34.

33. Goh VK, Lau C-P, Mohlenkamp S, et al. Outcome of coronary plaque burden: A 10-year follow-up of aggressive medical management. Cardiovasc Ultrasound 2010;8:5.

34. Anand DV, Lim E, Darko D, et al. Determinants of progression of coronary artery calcification in type 2 diabetes. Role of glycemic control and inflammatory/vascular calcification markers. J Am Coll Cardiol 2007;50:2218-25.

35. Burgstahler C, Reimann A, Beck T, et al. Influence of a lipid-lowering therapy on calcified and noncalcified coronary plaques monitored by multislice detector computed tomography: results of the New Age II Pilot Study. Invest Radiol 2007;42:189-95.

36. Mohler ER, Wang H, Medenilla E, et al. Effect of Statin treatment on aortic valve and coronary artery calcification. J Heart Valve Dis 2007;16:378-86

37. Budoff MJ, Yu D, Nasir K, et al. Diabetes and progression of coronary calcium under the influence of statin therapy. Am Heart J 2005;149:695700

38. Hecht HS, Harman SM. Evaluation by electron beam tomography of changes in calcified coronary plaque in treated and untreated asymptomatic patients and relation to serum lipid levels. Am J Cardiol 2003;91:1131-34.

39. Lo J, Lu MT, Ihenachor EJ, et al. Effects of statin therapy on coronary artery plaque volume and high-risk plaque morphology in HIV-infected patients with subclinical atherosclerosis: a randomised, double-blind, placebocontrolled trial. Lancet HIV 2015;2:e52-e63.

40. Zeb I, Li D, Nasir K, et al. Effect of statin treatment on coronary plaque progression - a serial coronary CT angiography study. Atherosclerosis 2013;231:198-204.

41. Hoffmann H, Frieler K, Schlattmann P, et al. Influence of statin treatment on coronary atherosclerosis visualised using multidetector computed tomography. Eur Radiol 2010;20:2824-33

42. Inoue K, Motoyama S, Sarai M, et al. Serial coronary CT angiographyverified changes in plaque characteristics as an end point: evaluation of effect of statin intervention. JACC Cardiovasc imaging 2010;3:691-98.

43. Auscher S, Heinsen L, Nieman K, et al. Effects of intensive lipid-lowering therapy on coronary plaques composition in patients with acute myocardial infarction: Assessment with serial coronary CT angiography. Atherosclerosis 2015;241:579-87.

44. Henein M, Granåsen G, Wiklund U, et al. High dose and long-term statin therapy accelerate coronary artery calcification. Int J Cardiol 2015;184:581-86

45. Nissen SE, Tuzcu EM, Schoenhagen P, et al. Effect of intensive compared with moderate lipid-lowering therapy on progression. JAMA 2004;291:1071-80.

46. Puri R, Libby P, Nissen SE, et al. Long-term effects of maximally intensive statin therapy on changes in coronary atheroma composition: Insights from SATURN. Eur Heart J Cardiovasc Imaging 2014;15:380-88.

47.Eshtehardi PP, McDaniel MC, Dhawan SS, et al. Effect of intensive atorvastatin therapy on coronary atherosclerosis progression, composition, arterial remodeling, and microvascular function. J Invasive Cardiol $2012 ; 24: 522$. 